\title{
Errors in juvenile copepod growth rate estimates are widespread: problems with the Moult Rate method
}

\author{
A. G. Hirst ${ }^{1, *}$, W. T. Peterson ${ }^{2}$, P. Rothery ${ }^{3}$ \\ ${ }^{1}$ British Antarctic Survey, Natural Environment Research Council, High Cross, Madingley Road, Cambridge CB3 0ET, UK \\ ${ }^{2}$ National Marine Fisheries Services, Hatfield Marine Science Center, 2030 S. Marine Science Drive, Newport, \\ Oregon 97365, USA \\ ${ }^{3}$ NERC Centre for Ecology and Hydrology, Monks Wood, Abbots Ripton, Huntingdon, Cambridgeshire PE28 2LS, UK
}

\begin{abstract}
The 'Moult Rate' (MR) method has been used widely to derive stage-specific growth rates in juvenile copepods. It is the most common field-based method. Unfortunately, the equation underlying the method is wrong and, consequently, large errors in juvenile growth rate estimates are widespread. The equation derives growth from the mean weight of 2 consecutive stages $(i$ and $i+1)$ and the duration of stage $i$. The weight change and the period to which this change is attributed are, therefore, offset. We explore this potential source of error in the MR method critically. Errors arise as a result of 2 primary factors: (1) unequal durations of successive stages and (2) unequal rates of growth of successive stages. The method of deriving the mean weight (arithmetic or geometric) also has an impact and is examined. Using a steady-state assumption, a range of scenarios and the errors that arise are examined. The literature is then reviewed and the size of errors resulting from MR method application in both field and laboratory situations is estimated. Our results suggest that the MR method can lead to large errors in growth estimation in any stage, but some stages are particularly prone. Errors for the C5 stage are often large because the following stage (the adult) does not moult, and has a different rate of body weight increase. For the same reason, errors are also great where the following stage is not actively moulting (e.g. when diapausing). In these circumstances, published work has commonly greatly underestimated growth. For example, MR growth ranges from 11 to $47 \%$ of the value derived correctly for this stage, $g_{i-c o r r}$ (calculated assuming the non-moulting stage does not grow). In late stages that are followed by actively moulting stages, the MR method has commonly given values in excess of $150 \%$ of $g_{i \_c o r r}$ but underestimation also occurs, with values $<90 \%$ of $g_{i \_c o r r}$. We propose new methods and equations that overcome these problems. These equations are written with and without within-stage mortality included. The equations are relatively insensitive to mortality rates within the range found in the field, but only provided that the stage duration is not determined from moult rate. Stage duration estimates obtained from measuring moulting rates of field-collected animals are very sensitive to mortality rates of the animals prior to capture, and field mortality rates are often high enough to produce dramatic over-estimation of stage duration.
\end{abstract}

KEY WORDS: Copepod $\cdot$ Moult Rate $\cdot$ Growth $\cdot$ Error $\cdot$ Stage duration $\cdot$ Mortality

Resale or republication not permitted without written consent of the publisher

\section{INTRODUCTION}

Copepods are the biomass-dominant mesozooplankton throughout the world's oceans (Verity \& Smetacek 1996). Their ubiquity has made them the focus of many studies that attempt to link pelagic primary production with higher trophic levels, and in budgets of pelagic carbon and nutrient flux. Only by quantifying the role of copepods can we fully understand the dynamics of pelagic marine systems (Banse 1995). An important aspect of both their population dynamics and their role in biogeochemistry is their rate of growth and sec- 
ondary production (the product of specific growth and biomass). This is why, over the last 3 decades, much effort has been expended in measuring near-natural rates of growth and development in copepods.

Historically, the most common method for examining 'growth' in copepods has been through the measurement of egg production rates, and derivation of weight-specific fecundity. However, we now have a clear understanding that egg production rates may not accurately reflect the growth of adults, because this stage can continue to add or lose weight whilst producing eggs (Hirst \& McKinnon 2001). Additionally, weight-specific fecundity rates are often dissimilar to juvenile somatic growth rates in nature; on average, the two diverge as temperature increases. The degree to which these two are food limited is also different, as is their variability (Hirst \& Bunker 2003). If we wish to have accurate measurements of copepod growth and production in future, we can no longer rely solely upon egg production rates. Juvenile growth rates must be examined directly, and more meticulously.

Of the various methods for measuring adult and juvenile growth, some are more direct than others, and assumptions vary, so some are more accurate than others. The Moult Rate method (MR method) is the most commonly applied approach for obtaining in situ juvenile growth rates. It is the main alternative to the 'Artificial Cohort' method (Kimmerer \& McKinnon 1987). Both methods can be used when natural cohorts are either not present or cannot be followed. Rates of moulting of a stage (i) are measured by incubating copepods of this stage over a fixed period, commonly $24 \mathrm{~h}$. Animals of stage $i$ and $i+1$ are also collected, and their mean weights measured. Alternatively, these are derived from lengths using length-weight equations (e.g. Peterson et al. 1991). Various equation forms have been used to describe weight-specific growth rates with the MR method (discussed later), but the most commonly applied is:

$$
g_{i_{-} M R}=\ln \left(\frac{W_{i+1}}{W_{i}}\right) \times M R_{i}
$$

Here, $g_{i-M R}$ is weight-specific growth of stage $i, W_{i}$ is mean weight of stage $i$, and $W_{i+1}$ is mean weight of stage $i+1 . M R_{i}$ is the moult rate $\left(\mathrm{d}^{-1}\right)$, i.e. the proportion of animals in stage $i$ moulting per day. The inverse of development time (i.e. $1 / D_{i}$ ) has, in many cases, been used rather than a measure of moult rate. These are equivalent when age within stage is uniform (i.e. there is zero mortality and the population is in steadystate). We return to this later to consider the case where mortality occurs ('Corrected equations' section). Use of Eq. (1) (or Eq. 1 with $M R_{\mathrm{i}}$ replaced with $1 / D_{i}$ ) is widespread, and it has been applied in obtaining stage-specific copepod growth rates in a large number of species in a wide range of environments, including in the studies of: Klein Breteler et al. (1982), Fransz \& Diel (1985), Diel \& Klein Breteler (1986), McLaren et al. (1989), Liang \& Uye (1991, 1996a,b), Peterson et al. (1991, 2002), Walker \& Peterson (1991), Hutchings et al. (1995), Webber \& Roff (1995), Liang et al. (1996), Hopcroft et al. (1998), Richardson \& Verheye (1998, 1999), Escribano \& McLaren (1999), Escribano \& Hidalgo (2000), Escribano et al. (2001), Richardson et al. (2001, 2003), Shreeve et al. (2002) and Rey-Rassat et al. (2004). It has also been used on zooplankton groups other than copepods (Muxagata et al. 2004). Jerling \& Wooldridge (1991) quote an equation where weights are described as being those at entry and exit from a stage; however, their actual application involved mean stage weights, but in this case of stage $i-1$ and stage $i$, their $M_{\min }$ and $M_{\max }$ respectively (H. Jerling pers. comm.).

Several workers have used an analogous method to derive growth rates $\left(g_{i \_L M R}\right)$ of animals (e.g. Burkill \& Kendall 1982, Uye et al. 1983, Kimoto et al. 1986, Huang et al. (1993), Kang \& Kang 1998). Instead of the exponential form described in Eq. (1), a linear equation form was used:

$$
g_{i_{-} L M R}=\left(\frac{W_{i+1}-W_{i}}{D_{i} \times W_{i}}\right)
$$

Here, $D_{i}$ is development time (in Burkill \& Kendall 1982 , this is obtained from $\left.D_{i}=1 / M R_{i}\right)$. This equation is freely convertible to the exponential form:

$$
g_{i_{-} M R}=g_{i_{-} L M R} \times\left(\frac{\ln W_{i+1}+\ln W_{i}}{W_{i+1}-W_{i}}\right) \times W_{i}
$$

The linear equation form still implicitly includes Assumptions 3 and 4 (described below), hence its inclusion here. Later, when we examine errors in the MR method, to allow ease of comparison in those studies using the linear equation form, we convert these results to the exponential form before determining the degree of error in these studies.

Our list of papers that apply the MR method is probably not exhaustive, but application spans $>20$ yr and at least 28 papers. These publications represent the majority of measurements we have on juvenile copepod growth rates, and the vast majority of stage-specific field rates. The accuracy of the method is, therefore, crucial; yet, as we shall demonstrate, it is seriously flawed, and at times has produced results that are in gross error.

\section{Assumptions}

The primary assumptions in the use of the MR growth equation are: (1) rate of weight increase is exponential over the period for which growth is calcu- 
lated, (2) age within stage is uniform (i.e. the population is in steady-state and mortality is zero), (3) durations of stages $i$ and $i+1$ are equal and (4) rates of growth in stages $i$ and $i+1$ are equal.

Assumptions 1 and 2 have to some degree been dealt with in the literature (see below). In some cases, these were not tested as a result of them impacting the MR method directly, but for other reasons. Assumptions 3 and 4, however, have gone largely missed or ignored. Assumption 1, that growth is exponential in form between the 2 time points, has been examined often, and at various time/stage scales (e.g. Miller et al. 1977, Huntley \& Lopez 1992 and Escribano \& McLaren 1992). At the level of egg to adult, body weight often increases in a sigmoid fashion in copepods (Vidal 1980, A. Hirst pers. obs.), and there is evidence that growth can vary from stage to stage. Assumption 2 has been examined previously by Miller et al. (1984) and Miller $\&$ Tande (1993). The primary reason for the exploration of age within stage is that if age is not uniform, then development time is not correctly assessed from experimental determination of the moulting rate (i.e. $1 / M R_{i} \neq$ $\left.D_{i}\right)$. There is a second reason an assumption of uniform age within stage is critical to the MR method. If age within stage is non-uniform, then the mean weight ( $W_{i}$ and $W_{i+1}$ ) will also be biased. Here, we assume that the population is in steady-state (e.g. there is no cohort structure moving through the stages). If violated, this assumption can have important consequences, but this is outside what we wish to focus our attention on here. Initially, when we explore error in the MR method, we assume mortality to be zero or negligible. Later, in the 'Corrected equations' section, we deal with the situation where mortality is not zero. The impact of mortality on moult rate (stage duration), mean weight and growth rates are all examined, and equations that allow for mortality are derived. Whether geometric or arithmetic mean weights are applied to stages impacts the degree of error obtained; we therefore consider both averaging forms. Geometric mean weights of a stage are difficult to determine practically because weighing often involves the bulking of animals. By contrast, arithmetic mean weight can be determined even if animals are bulked together.

Assumptions 3 and 4 arise because growth is a measure of weight increment over a specific period, yet the MR equation does not attribute the correct period of time to the weight increase. This point is examined in Fig. 1. The shaded areas demonstrate both the period over which the weight increment is attributed (i.e. the stage duration), and the points from which the start and end weights are taken in determining growth. Let us start by comparing growth derived for a stage $i$ using Eq. (1), with the correct definition of growth in this stage, which is given as:

$$
g_{i_{-} \text {corr }}=\ln \left(\frac{W_{i \_ \text {exit }}}{W_{i_{-} \text {entry }}}\right) \times M R_{i}
$$

In this equation, growth is determined from the point of entry to the stage, $W_{i_{-} \text {entry }}$ and at exit, $W_{i \_ \text {exit }}$ (these are arithmetic mean weights of animals entering and leaving stage $i$ ). The weight at exit should include the exoskeleton if the animal has just moulted out of the stage. This equation is the $g_{\text {corr }}$ term we refer to throughout the paper.

In Fig. 1a, there is no difference between the growth rate of stage $i$ derived using the MR growth equation for $g_{i \_M R}$, and that derived more correctly from the weight at the point of entry and exit from stage $i\left(g_{i_{-} \text {corr }}\right)$. However, in Fig. 1 b, the rates of weight increment in stages $i$ and $i+1$ are equal, but the stage durations are not the same (Assumption 3 is violated). Consequently, estimates of growth from $g_{i_{-} M R}$ are in error, and these differ from $g_{i_{-} \text {corr }}$. In Fig. 1c, the duration of stages $i$ and $i+1$ are equal, but the rate of weight acclimation differs (Assumption 4 is violated). Again, $g_{i_{-M R}}$ produces erroneous results. These simple examples also highlight how the method by which mean weight is obtained (simple arithmetic or geometric) also impacts the $g_{i_{-} M R}$ value and the degree of error (see Fig. 1b,c).

The aims of this study are to demonstrate where, when and why the MR method produces erroneous growth values as a result of violation of Assumptions 3 and 4 . These assumptions have been virtually ignored, but they are critical. Initially, we focus on examining the potential size of error under a range of possible circumstances ('Simulations of errors' section). We then describe errors in published growth rates ('Assessing error in field and laboratory data'). Equations and descriptions for a new method are finally described ('Corrected equations'), including considerations of the impacts of within-stage mortality.

\section{SIMULATIONS OF ERRORS}

When assessing error in $g_{i_{-} M R}$ rates, we compare these values directly with $g_{i_{\text {c corr }}}$ values (see Fig. 1). We express the former as a percentage of the latter; hence, a value of $100 \%$ occurs when the MR method gives the correct result.

Errors due to violation of Assumptions 3 and 4 are examined in turn; we assume mortality to be zero. Firstly, growth rates of stages $i$ and $i+1$ are set as equal, and error in $g_{i_{-} M R}$ resulting from different stage durations in $i$ and $i+1$ is examined. Secondly, stage durations of $i$ and $i+1$ are set as equal, and error in $g_{i_{-} M R}$ resulting from different values of $g_{i_{-} \text {corr }}$ in the 2 stages is examined. If weight is derived using geomet- 

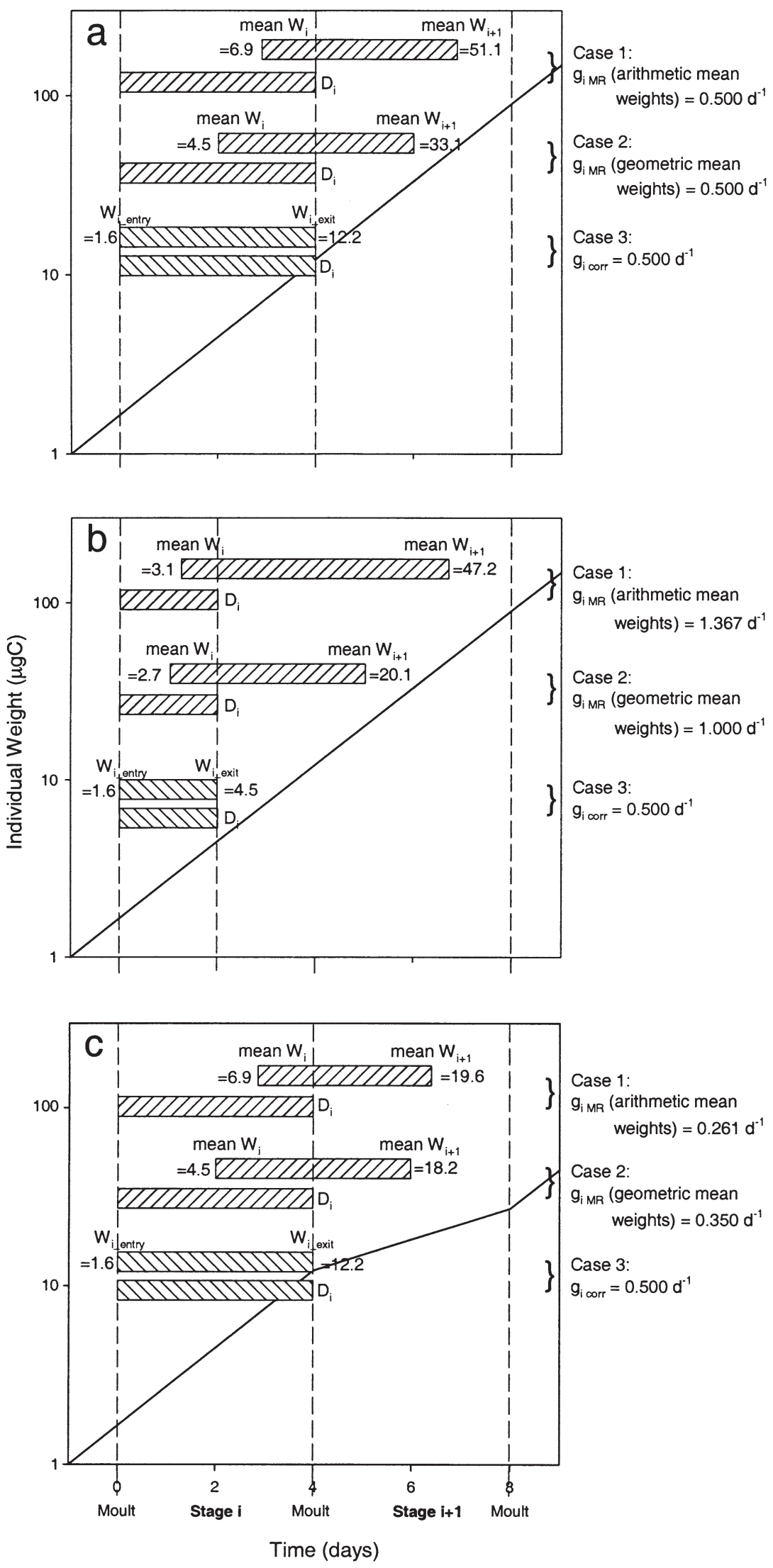

ric means, the size of the error is not sensitive to the size of $g_{i_{-} \text {corr }}$. However, when arithmetic mean weights are used to derived $g_{i \_M R}$, the size of the error is dependent upon the absolute rate of $g_{i_{i} \text { corr }}$. To explore this, we derived error in $g_{i_{-} M R}$ using a range of $g_{i_{-} c o r r}$ values, $0.02,0.2$ and $2.0 \mathrm{~d}^{-1}$. The extremes of these values were chosen so as to represent the range of most juvenile copepod growth rates (Hirst \& Bunker 2003). As the degree of error is sensitive to the absolute stage duration when arithmetic mean weights are used, error was derived for 2 stage duration values $\left(D_{i}\right), 1$ and $10 \mathrm{~d}$ (these are presented in Fig. 2a,b, respectively). Note that errors in $g_{i_{-} M R}$ derived using geometric mean weights are not sensitive to the absolute value of $D_{i}$ (hence, in Fig. 2a, b, the error is the same for the $g_{i_{-} M R}$ derived using geometric mean weights).

The greater the divergence from unity in the ratio of successive stage durations $\left(D_{i} / D_{i+1}\right)$, the greater the error in $g_{i \_M R}$. When geometric mean weights are used, if the older stage has a shorter duration $\left(D_{i} / D_{i+1}>1\right)$ but the same rate of weight increase, then $g_{i_{-} M R}$ underestimates the correct rate of growth by a maximum of $50 \%$ of the true value $\left(g_{i_{-} \text {corr }}\right)$. However, when arithmetic mean weights are used,

Fig. 1. Examples of the application of the Moult Rate (MR) method to derive growth $\left(g_{i \_M R}\right)$ in stage $i$, and the correct growth rate $\left(g_{i_{-} \text {corr }}\right)$. Shaded areas demonstrate the period over which development time and weight changes are applied to derive the growth rate. Note that when using the MR method, these are offset. The weight trajectory of animals as they pass through the various stages is given by the bold line. Case 1 examples are where the MR method is used and the arithmetic mean weights of consecutive stages are applied. Case 2 examples are where the MR method is used and geometric mean weights of consecutive stages are applied. Case 3 examples are where the growth of stage $i$ is correctly determined from $D_{i}$ and the changes in weight over the same period. (a) Stages $i$ and $i+$ 1 have equal duration and rate of daily growth. In this instance, when $g_{i_{-} M R}$ and $g_{i_{i} \text { corr }}$ are applied to stage $i$, results for both are equal. (b) Stages $i$ and $i+1$ have unequal duration but equal rates of daily growth. In this instance, $g_{i \_M R}$ is incorrect (i.e. not equal to $g_{i_{-} \text {corr }}$ ), and Assumption 3 is violated. (c) Stages $i$ and $i+1$ have equal duration but unequal rates of daily growth. In this instance, $g_{i_{-} M R}$ is incorrect (i.e. not equal to $\left.g_{i \_c o r r}\right)$, and Assumption 4 is violated 
the degree to which growth can be underestimated using the MR method can be greater, with larger $g_{i_{-} \text {corr }}$ and larger $D_{i}$ values leading to greater underestimation. If the older stage has a longer duration $\left(D_{i} / D_{i+1}<\right.$ 1), growth rates will be overestimated using the MR method. In this instance, there is no absolute limit to the degree to which the MR method can overestimate,

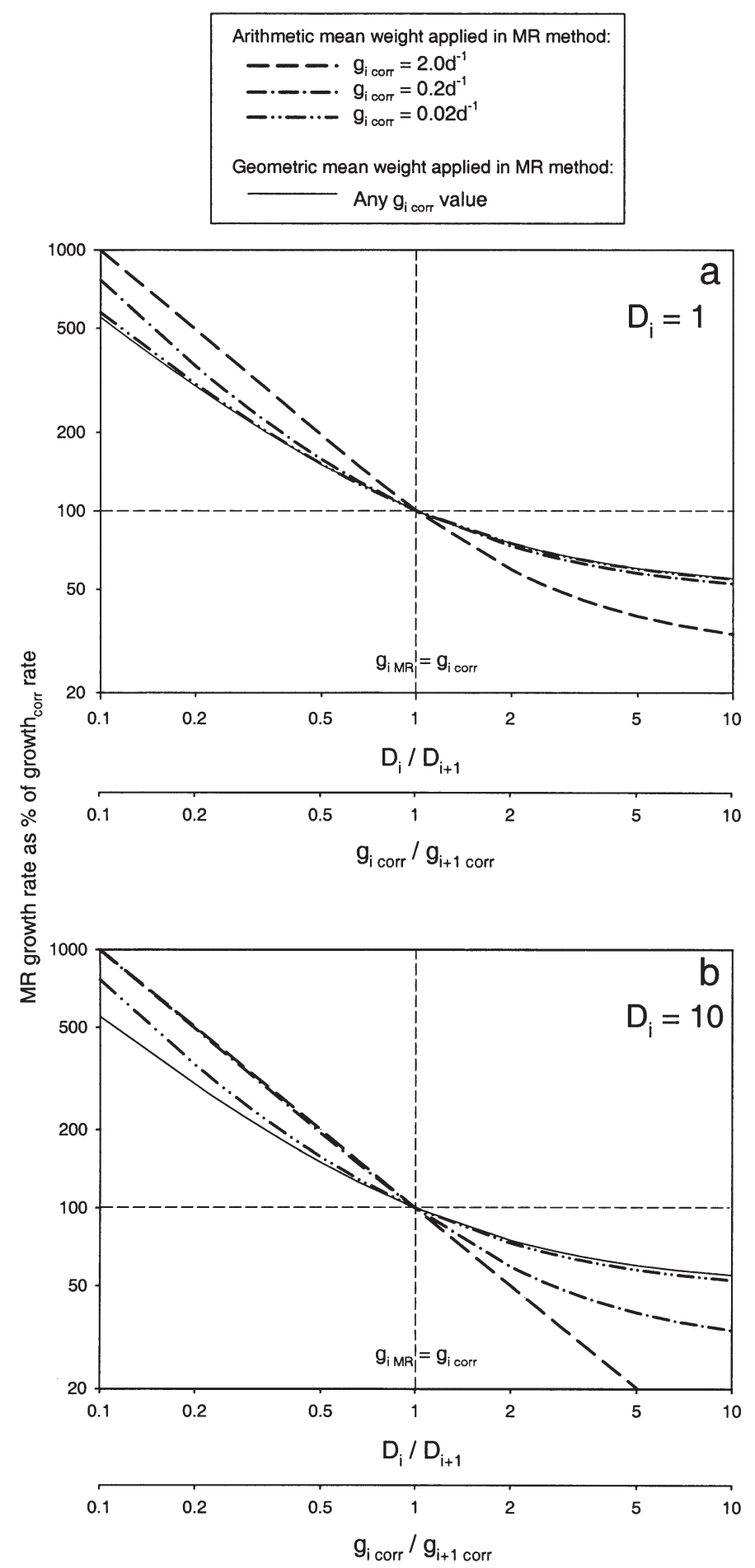

but to give an example, if $D_{i} / D_{i+1}$ is 0.1 , and geometric mean weights are used, then the $g_{i \_M R}$ will be 5.5 times $g_{i_{-} \text {corr }}$ (i.e. $550 \%$ of the correct value). In these examples, equal ratios of $D_{i} / D_{i+1}$ and $g_{i \_ \text {corr }} / g_{i+1 \_ \text {corr }}$ give equal errors. The absolute rate of $g_{i \_c o r r}$ and duration of stage $i\left(D_{i}\right)$ does not alter the percentage that the MR growth rate is in error when geometric mean stage weights have been applied. In these examples, when the ratios of $D_{i} / D_{i+1}$ or $g_{i \_c o r r} / g_{i+1 \_c o r r}$ are 1, Assumptions 3 and 4 are both met (this is because our method of analysis was to set the other unexamined ratio in these examples to be equal, i.e. 1), and therefore the MR method gives the correct growth values.

In order to determine which stages will be most vulnerable to the errors examined in the simulations, we compiled data on the duration of successive stages in a range of planktonic copepods, i.e. $D_{i}$ and $D_{i+1}$ (values from: Hart 1990, Uye et al. 1983, Kimoto et al. 1986, Fryd et al. 1991, Kang \& Kang 1998), and on the growth rate of the successive stage, i.e. $g_{i_{-} \text {corr }}$ and $g_{i+1_{1} \text { corr }}$ (values from: Shreeve \& Ward 1998, Rey-Rassat et al. 2002). These data are presented in Fig. $3 a, b$ as ratios for successive stages $\left(D_{i} / D_{i+1}\right.$ and $\left.g_{i \_c o r r} / g_{i+1 \_c o r r}\right)$. There are commonly radical differences in development times between the stages at N2 to N3, N3 to N4, N6 to C1 and C4 to C5 (Fig. 3). Non-isochronal stage durations are obvious in many genera, e.g. Calanoides, Calanus, Paracalanus, Pseudocalanus, Pseudodiaptomus, Rhincalanus and Temora (Hart 1990, Peterson 2001), with stage durations generally declining from CI onwards. It is common for the ratio of $D_{i} / D_{i+1}$ to be greater than 2 or less than 0.5 (Fig. 3a). Although some copepod species may come close to having isochronal stage durations (e.g. Acartia), the majority diverge widely (Hart 1990, Peterson 2001). This clearly has important conse-

Fig. 2. Examination of error using the Moult Rate (MR) method under different scenarios of growth and development times. (a) Upper $x$-axis: MR growth $\left(g_{i_{-} M R}\right)$ of stage $i$ expressed as a percentage of the correct growth rate $\left(g_{i_{-} \text {corr }}\right)$ plotted against the relative stage duration of stage $i$ to that of stage $i+1\left(D_{i} / D_{i+1}\right)$, and derived for 3 different rates of correct growth, $g_{\text {corr }}=2.0,0.2$ and $0.02 \mathrm{~d}^{-1}$. Note that the absolute rate of growth is only of consequence if the arithmetic mean of weight has been applied rather than the geometric mean weight. In this example, the growth rate $\left(g_{\text {i_corr }}\left[\mathrm{d}^{-1}\right]\right)$ is assumed equal in stages $i$ and $i+1$. Lower $x$-axis: MR growth $\left(g_{i_{-} M R}\right)$ of stage $i$ expressed as a percentage of the correct growth rate $\left(g_{i_{i} \text { corr }}\right)$ plotted against the relative growth rates of stage $i$ to that of stage $i+1\left(g_{i_{-} c o r r} / g_{i+1}\right.$ corr $)$, and derived for 3 different rates of correct growth $\left(g_{i_{\text {_corr }}}=2.0,0.2\right.$ and $\left.0.02 \mathrm{~d}^{-1}\right)$. In this example, the development time ( $D[\mathrm{~d}])$ is assumed equal in stages $i$ and $i+1$. Stage duration of $i$ is set at $1 \mathrm{~d}$. (b) As in upper panel, but with stage duration of $i$ set at $10 \mathrm{~d}$. Note from both panels that the absolute rate of $g_{i_{-} c o r r}$ and the duration of stage $i$ are only of consequence to the degree of error if the arithmetic mean weight has been applied rather than the geometric mean weight 

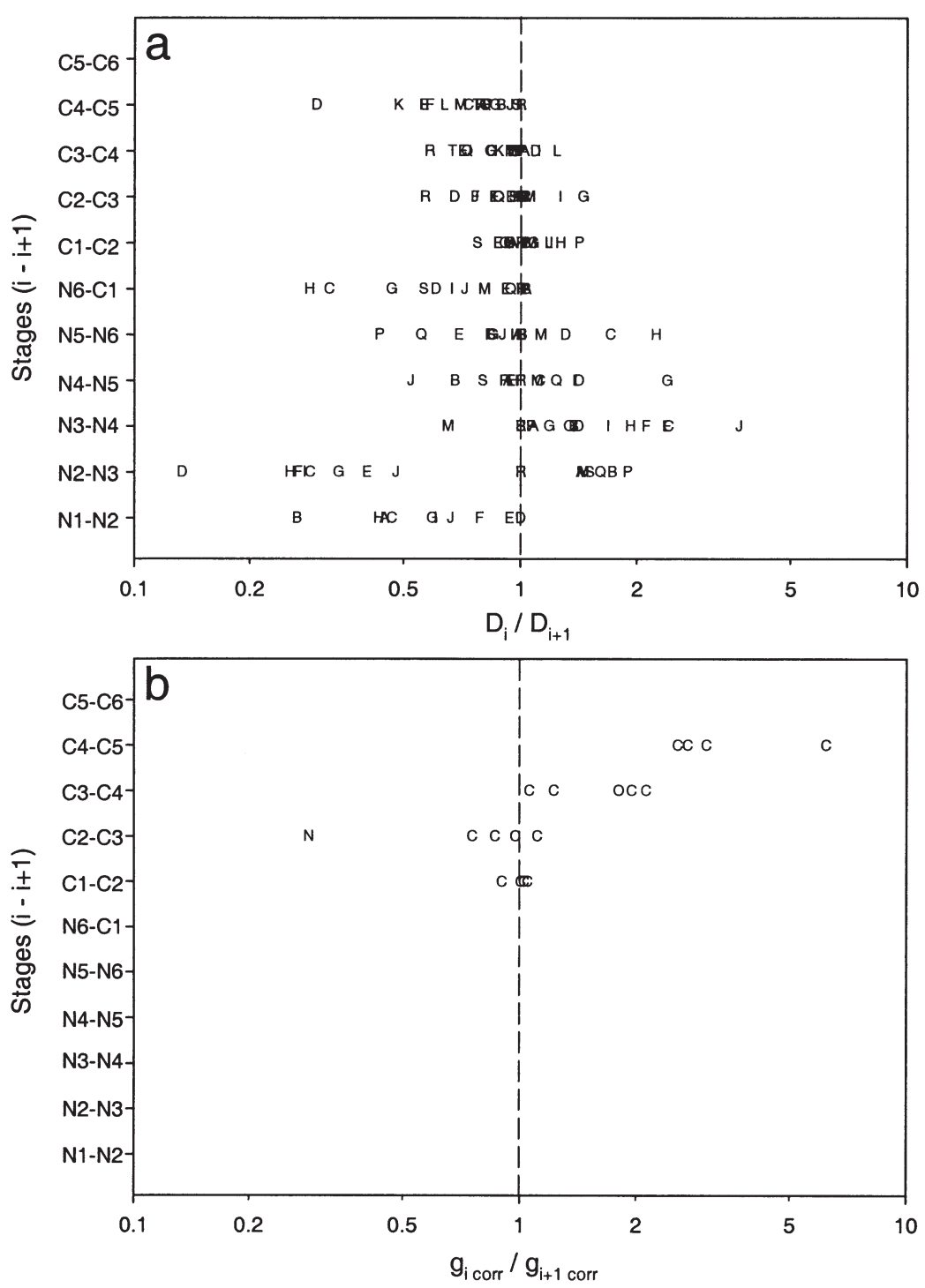

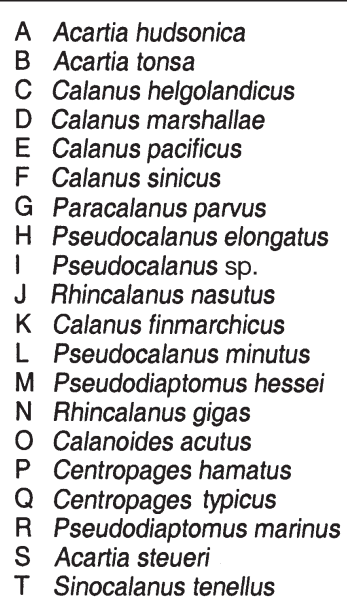

Fig. 3. Compilation of literature values of stage durations and growth rates of successive stages. (a) Values of $D_{i} / D_{i+1}$ for development stages N1-N2 through to C5-C6 for a variety of marine planktonic copepods. (b) Values of $g_{i} / g_{i+1}$ for the development stages N1-N2 to C5-C6 for a variety of marine planktonic copepods. Development and growth data are taken from the published literature. See text for details quences on the size of error resulting from application of the MR method. Ratios of $g_{i_{-} \text {corr }} / g_{i+1}$ corr also diverge from 1. Comparing these ratios from real examples with the simulated errors detailed in Fig. 2, suggests that not only will errors frequently occur but that they are likely to be substantial.

A special problem arises for copepodite Stage 5, and for any other stage that is followed by one which does not moult (e.g. when preparing for or in diapause). Stages that do not moult can commonly gain or lose weight (e.g. Durbin et al. 1992, McKinnon 1996, Hirst \& McKinnon 2001). We examine error in the MR results for these situations when mean weight achieved by stage $i+1$ varies from $50 \%$ of that at which it entered the stage $\left(W_{i_{-} \text {exit }}\right)$, to where it is $150 \%$ of that at entry. This range in weight increase in a non-moulting stage encompasses the majority of weight changes that have been found in adult copepods (Hirst \& McKinnon 2001). As the error is sensitive to the size of $g_{i_{-} \text {corr }}$ and $D_{i}$, we have derived the error for $g_{i_{-} \text {corr }}$ values of 0.02 , 0.2 and $2.0 \mathrm{~d}^{-1}$, and for $D_{i}$ values of 1,5 and $10 \mathrm{~d}$ (Fig. 4). Regardless of whether the non-moulting stage gains, loses or stays the same weight, errors can occur. The $g_{i_{-} M R}$ value is almost always a poor representation of the correct value, always underestimating $g_{i \_c o r r}$ when the mean weight of the non-moulting stage is lower than the weight at entry, and often underestimating when $g_{i_{-} c o r r}>0.2 \mathrm{~d}^{-1}$. To date, late copepodite stages have been those principally examined using the MR method. Many of the published studies, therefore, contain a sizeable proportion of $g_{M R}$ rates for stages that are followed by a non-moulting stage, and errors of this particular form are common. Errors in published studies are examined in more detail below. 


\section{Assessing error in field and laboratory data}

We attempt to assess the degree of error in a range of published studies that have used the MR method to determine growth rates. We explore errors in the $g_{i \_M R}$ values of 13 published studies (18 species). Table 1 details the equations used in these original studies, sources of stage duration in these papers, and the form that mean weight took. In all cases, arithmetic mean weight was used, except in one case, in which the arithmetic mean length was converted to a weight. In all cases, mortality was assumed to be zero. When the linear form of MR growth rates $\left(g_{i_{-} L M R}\right)$ had been applied, we examined the degree of error after this had been corrected to the exponential form.

First, let us detail how error is derived in stage $i$ where the following stage $(i+1)$ moults. There is not sufficient information to account for the error arising in $g_{M R}$ estimates from both different growth rates in successive stages, and also for differences in stage duration. There is also not the data necessary to correctly determine $g_{i \text { corr }}$ and $g_{i+1 \_c o r r}$. In order to attempt to estimate error, we therefore assumed $g_{\text {corr }}$ to be equal in stages $i$ and $i+1$. We know this assumption will not be correct in many cases, but our estimates should still give us a first approximation as to the size of errors in the literature. To determine error, the following model approach was adopted. Animals were grown at $3 g_{\text {corr }}$ values $\left(0.02,0.2\right.$ and $\left.2.0 \mathrm{~d}^{-1}\right)$ through the stage pairs, the duration of each of the stages having been taken from the original publication being examined (see Table 1 for sources). These $g_{\text {corr }}$ values were chosen to encompass the vast majority of juvenile rates. A range was used as the size of the error is sensitive to the absolute value of $g_{\text {corr }}$. From these models, the arithmetic mean weights of the animals were determined for stages $i$ and $i+1$, and Eq. (1) was used to derive MR growth rate. Finally, this value could be compared against our known $g_{\text {corr }}$ values, and the degree of error assigned. This method was repeated for every stage pair for which we had appropriate laboratory or field data.

Next, we determined the error in $g_{i-M R}$ for the C5 stage, and in other stages that are followed by a nonmoulting stage. Three scenarios were examined: (1) when the non-moulting stage achieves a mean weight that is $50 \%$ greater than its weight at entry, $(2)$ when the non-moulting stage $(i+1)$ achieved a mean weight equal to that at the point of moulting into the stage, and (3) when the mean weight is $50 \%$ less than that at entry (to give an example, an animal weighing $10 \mathrm{mg} \mathrm{C}$ ind. $^{-1}$ at point of entry that loses $50 \%$ of its weight, achieves a mean weight of $5 \mathrm{mg} \mathrm{C}$ ind. ${ }^{-1}$ ). Again, we derived error for various $g_{i \text { corr }}$ values: $0.02,0.2$ and $2.0 \mathrm{~d}^{-1}$. Jerling \& Wooldridge (1991)
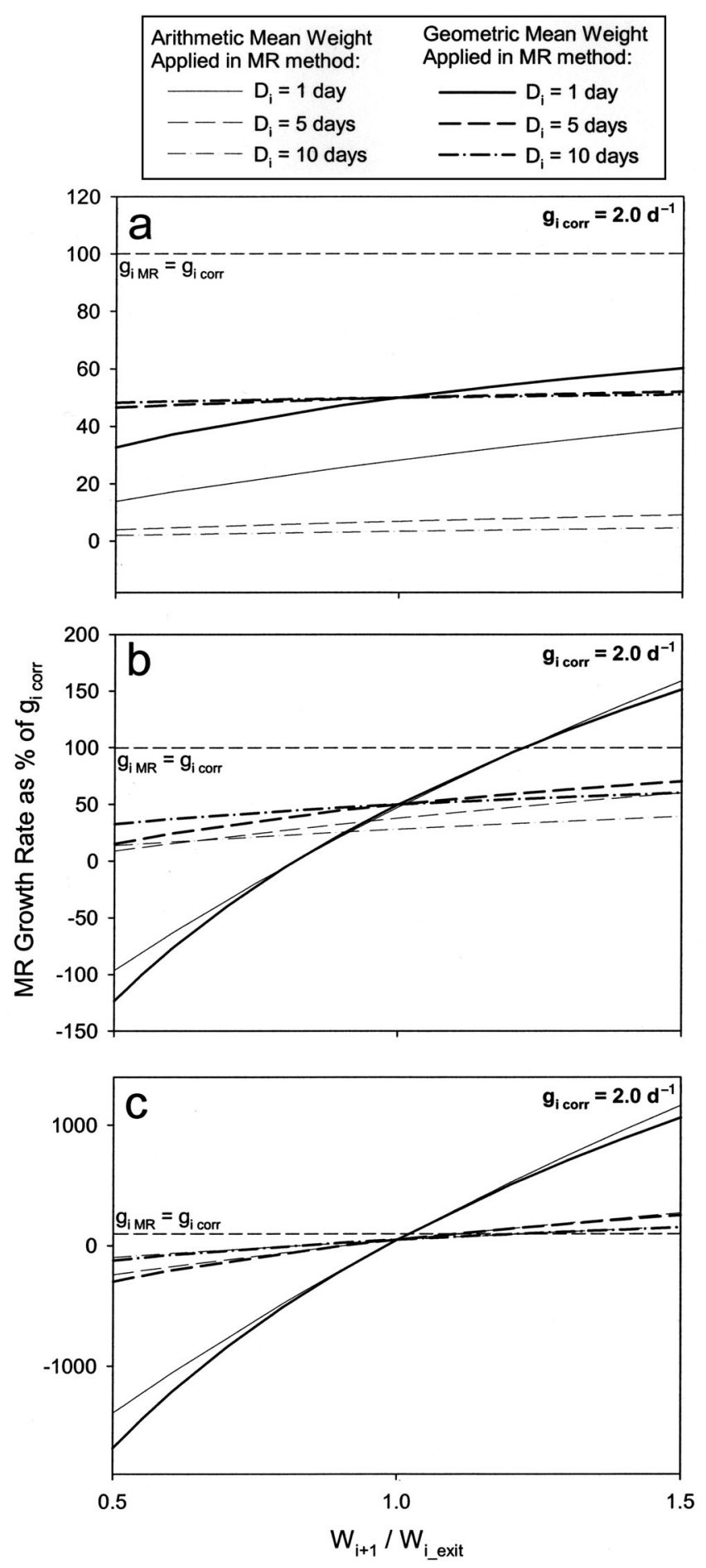

Fig. 4. Examination of errors associated with the application of the Moult Rate (MR) method to derive growth of stage $i$ when the following stage $(i+1)$ is a non-moulting stage. Error was examined as a function of the ratio of the mean weight achieved by the non-moulting stage $\left(W_{i+1}\right)$ divided by the weight at the point of stage entry $\left(W_{i_{-} \text {exit }}\right)$. Error derived when: (a) $g_{i \_c o r r}$ is $2.0 \mathrm{~d}^{-1}$, (b) $0.2 \mathrm{~d}^{-1}$ and (c) $0.02 \mathrm{~d}^{-1}$. Short dashed lines indicate where $g_{i_{-} M R}=g_{i_{-} \text {corr }}$, i.e. where growth rate derived by the MR method is correct 
Table 1. Studies in which the Moult Rate method is used. These examples are examined with respect to error arising from unequal stage duration $(i$ and $i+1)$. Fig. 5 examines the in situ studies, and Fig. 6 the laboratory studies. Eq. (1) is: $g_{i \_} M R=\ln$ $\left(W_{i+1} / W_{i}\right) \times M R_{i}$. Eq. $\left(1^{*}\right)$ is a slightly different version of Eq. (1), given as: $g_{i-M R}=\ln \left(W_{i} / W_{i-1}\right) \times M R_{i}$. Eq. $(2)$ is: $g_{i L M R}=$ $\left(W_{i+1}-W_{i}\right) /\left(W_{i} \times D_{i}\right)$. Durations of stages to derive errors from are taken from the tables in the original studies as detailed below. Methods used to derive mean stage weights were provided as a personal communication from the first or second author

\begin{tabular}{|c|c|c|c|c|c|}
\hline Species & $\begin{array}{l}\text { Growth } \\
\text { equation }\end{array}$ & $\begin{array}{l}\text { Method of mean } \\
\text { stage weight }\end{array}$ & $\begin{array}{l}\text { Source of stage } \\
\text { duration }\end{array}$ & $\begin{array}{l}\text { Figure in } \\
\text { this paper }\end{array}$ & Source \\
\hline \multicolumn{6}{|l|}{ In situ: } \\
\hline Calanus agulhensis & 1 & Arithmetic weight & Tables V; 1 & $5 a$ & Richardson \& Verheye $(1998,1999)$ \\
\hline Calanus chilensis & 1 & Arithmetic weight & Table 1 & $5 \mathrm{n}$ & Escribano \& McLaren (1999) \\
\hline Calanus finmarchicus & 1 & Arithmetic weight & Averages in text & $5 \mathrm{c}$ & Peterson et al. (1991) \\
\hline Calanus marshallae & 1 & Arithmetic weight & Table 2 & $5 d$ & Peterson et al. (2002) \\
\hline Calanoides acutus & 1 & Arithmetic weight & Table 1 & $5 e$ & Shreeve et al. (2002) \\
\hline Calanoides carinatus & 1 & Arithmetic weight & Table V & $5 b$ & Richardson \& Verheye (1998) \\
\hline Centropages velificatus & 1 & Arithmetic length $^{\mathrm{a}}$ & Table 2 & $5 \mathrm{k}$ & Hopcroft et al. (1998) \\
\hline Euchaeta marina & 1 & Arithmetic weight & Table 4 & $5 \mathrm{i}$ & Webber \& Roff (1995) \\
\hline Eurytemora affinis & 2 & Arithmetic weight & Table 4 (24 January) & $5 \mathrm{~m}$ & Burkill \& Kendall (1982) \\
\hline Oithona plumifera & 1 & Arithmetic weight & Table 4 & $5 \mathrm{j}$ & Webber \& Roff (1995) \\
\hline Paracalanus/Clausocalanus spp. & 1 & Arithmetic weight & Table 4 & $5 \mathrm{~g}$ & Webber \& Roff (1995) \\
\hline Pseudodiaptomus hessei & $1^{*}$ & Arithmetic weight & Table $2\left(16^{\circ} \mathrm{C}\right)$ & 50 & Jerling \& Wooldridge (1991) \\
\hline Rhincalanus gigas & 1 & Arithmetic weight & Table 1 & $5 \mathrm{f}$ & Shreeve et al. (2002) \\
\hline Temora turbinata & 1 & Arithmetic length $^{\mathrm{a}}$ & Table 2 & 51 & Hopcroft et al. (1998) \\
\hline Undinula vulgaris & 1 & Arithmetic weight & Table 4 & $5 \mathrm{~h}$ & Webber \& Roff (1995) \\
\hline \multicolumn{6}{|l|}{ Laboratory: } \\
\hline Acartia steueri & 2 & Arithmetic weight & Table 4 & $6 c$ & Kang \& Kang (1998) \\
\hline Pseudodiaptomus marinus & 2 & Arithmetic weight & Table 1 & $6 b$ & Uye et al. (1983) \\
\hline Sinocalanus tenellus & 2 & Arithmetic weight & Table 4 & $6 a$ & Kimoto et al. (1986) \\
\hline
\end{tabular}

did not use C5 and C6 mean weights when determining growth in C5, but rather C4 and C5 weights (see our Introduction), so we apply appropriate equations to these stages (Fig. 5o).

In our assessment of the error arising from unequal stage duration (Figs. $5 \& 6$ ), but where both stages are moulting, we find on several occasions $g_{i \_M R}$ values for these stages to be in excess of $200 \%$ of $g_{i_{-} \text {corr }}$ i.e., values are over twice what they should be (e.g. Calanus agulhensis and Calanoides carinatus, Richardson \& Verheye 1998, 1999; Acartia steueri, Kang \& Kang 1998; Calanus finmarchicus, Peterson et al. 1991). While values are commonly greater than $150 \%$ (e.g. Calanoides acutus and Rhincalanus gigas, Shreeve et al. 2002; Calanus agulhensis and Calanoides carinatus, Richardson \& Verheye 1998, 1999; Calanus chilensis, Escribano \& McLaren 1999; Acartia steueri, Kang \& Kang 1998; Calanus finmarchicus, Peterson et al. 1991; Pseudodiaptomus marinus, Uye et al. 1983; Eurytemora affinis, Burkill \& Kendall 1982), underestimation also occurs, with $g_{i_{-} M R}$ values at times being 80 to $90 \%$ of $g_{i \_c o r r}$ (e.g. Calanoides carinatus, Richardson \& Verheye 1998; Centropages velificatus, Hopcroft et al. 1998; Pseudodiaptomus hessei, Jerling \& Wooldridge 1991; Acartia steueri, Kang \& Kang 1998).

In stages followed by a moulting stage, the MR method has tended to overestimate the correct growth rate (Figs. $5 \& 6$ ). This is because, in most of these studies, copepodite stages have been examined (presumably because these are easier to work on, or they dominate the species biomass), and stage duration typically increases stage on stage in copepodites (i.e. $D_{i} / D_{i+1}<1$, see Fig. 3). For any given $D_{i} / D_{i+1}$ value, longer stage durations give greater errors than for short stage durations (compare $D_{i}=1$ and $10 \mathrm{~d}$ in Fig. 2). However, the error is only greater where arithmetic mean weights are applied, and not for geometric weights. These observations help explain why many of the studies on large bodied copepods in colder waters show greater errors than studies of small species in warm water; the former tend to have longer stage durations. For nauplii, based on differences in stage duration alone, the MR method would tend to overestimate growth for N1 $\left(D_{i} / D_{i+1}\right.$ is commonly $<1$, Fig. 3$)$. As $D_{i} / D_{i+1}$ is very variable from species to species for the N2-N3 pair, error would also be very variable in the N2 stage. These predictions are borne out by the few laboratory studies of naupliar MR growth. N1 growth is dramatically overestimated for Acartia steueri (Fig. 6c), while N2 growth has been both over- and underestimated (Fig. 6a,c).

When we assume that adults do not grow after the point of moulting, and $g_{i_{-} \text {corr }}$ of copepodite Stage 5 is taken as $0.2 \mathrm{~d}^{-1}$, estimates of errors in published studies give $g_{i \_M R}$ values for Stage 5 copepodites as being 

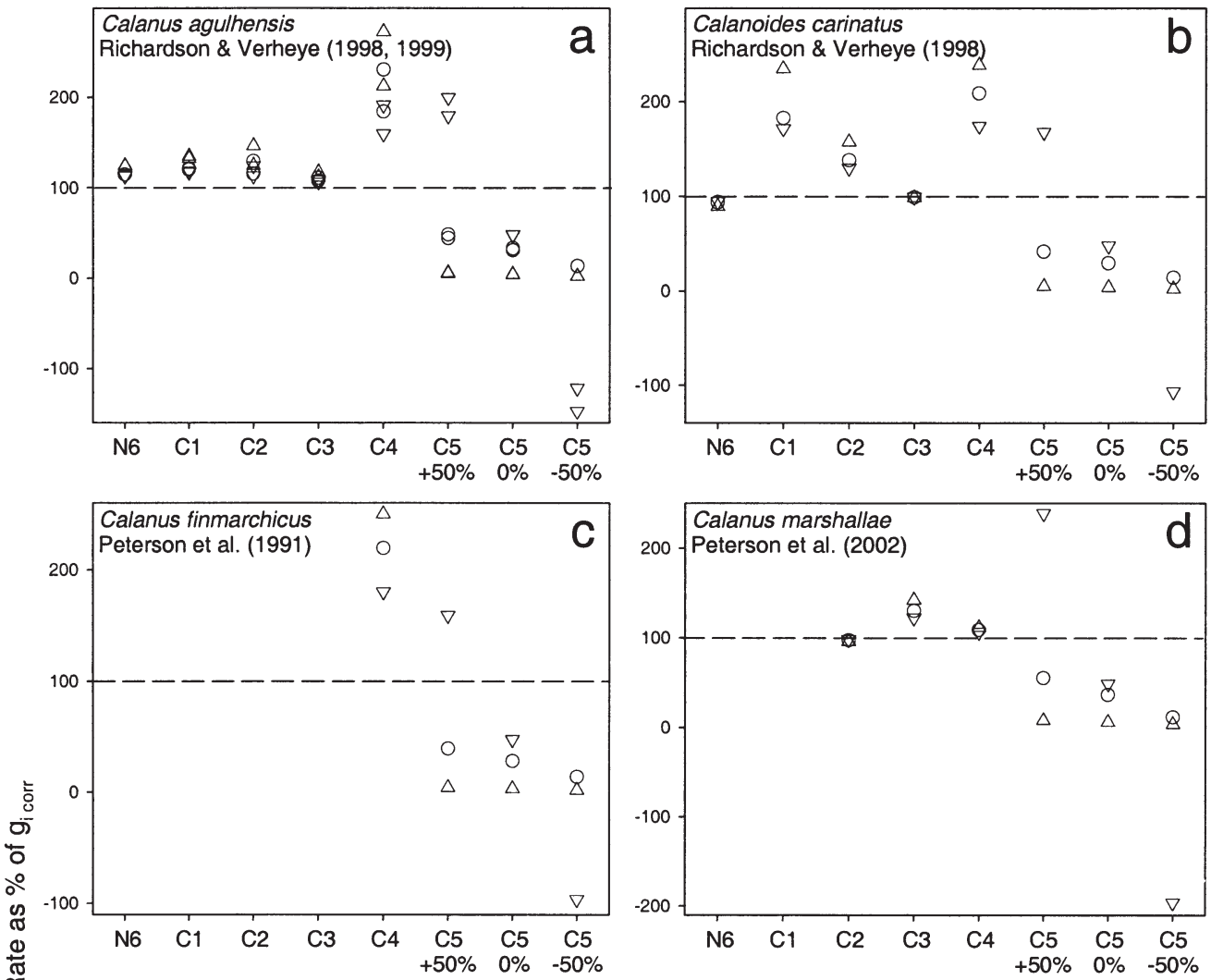

莫

e
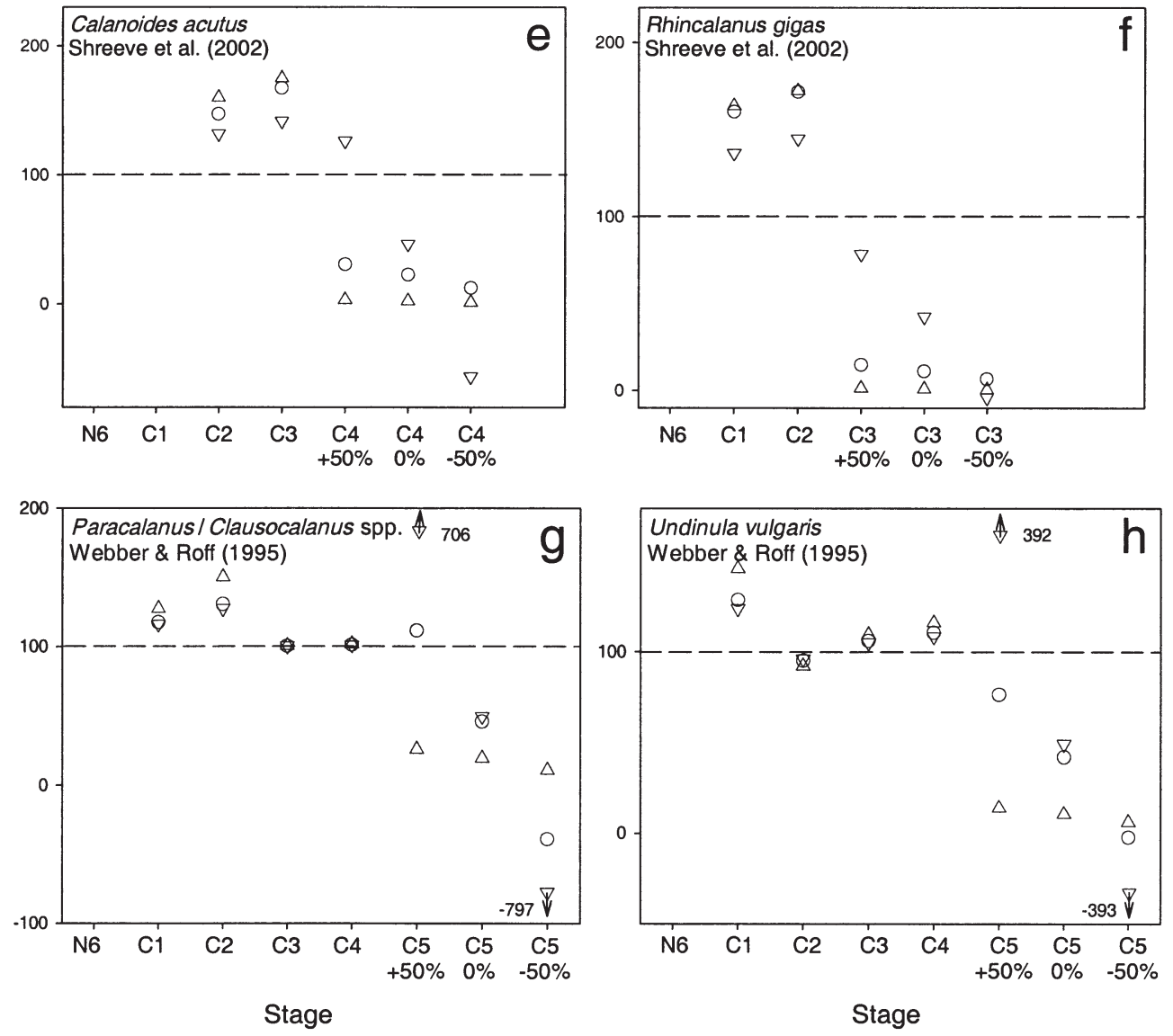

Fig. 5. (Above and following page) 

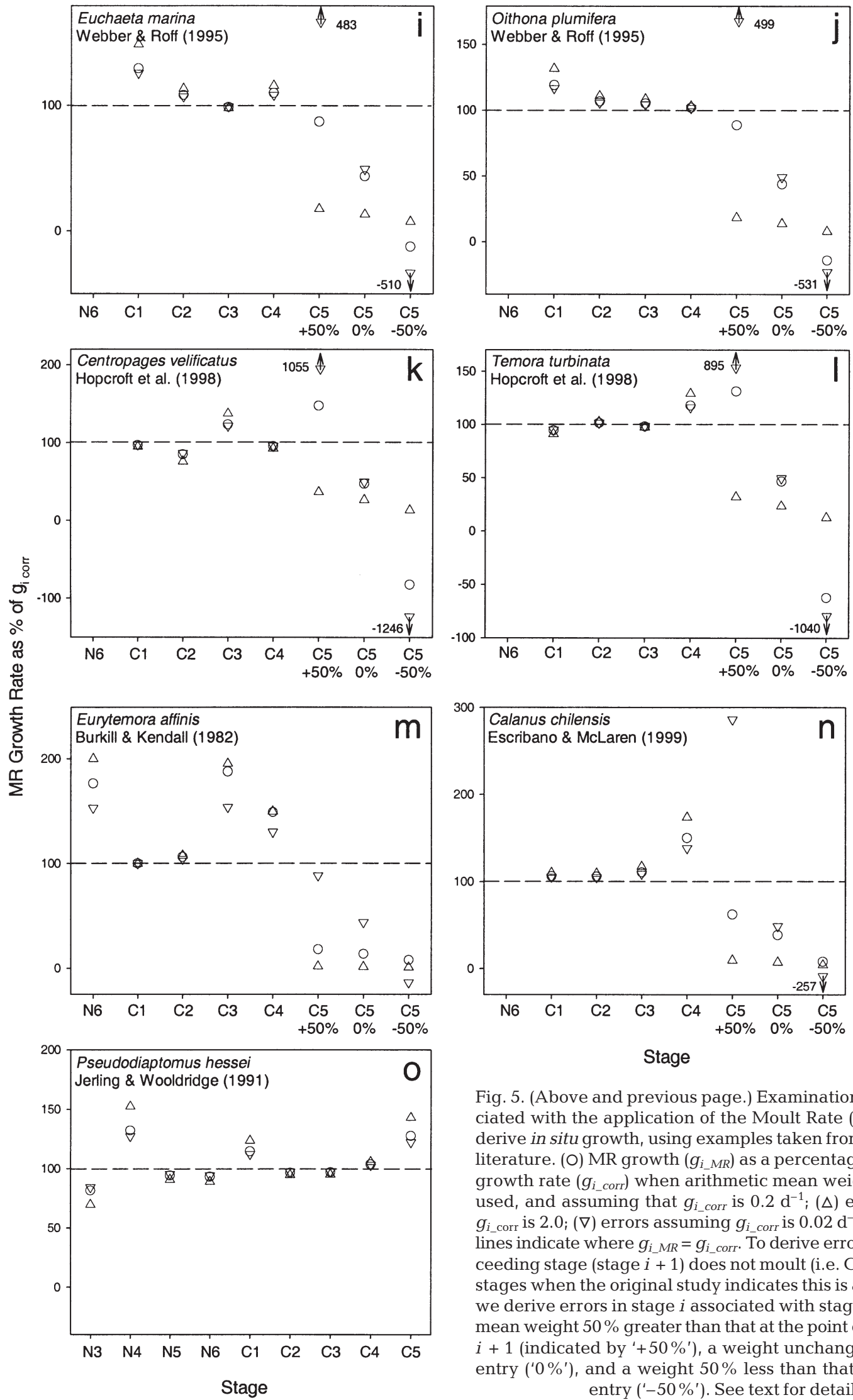

Fig. 5. (Above and previous page.) Examination of errors associated with the application of the Moult Rate (MR) method to derive in situ growth, using examples taken from the published literature. (O) MR growth $\left(g_{i_{-} M R}\right)$ as a percentage of the correct growth rate $\left(g_{i_{\text {ccorr }}}\right)$ when arithmetic mean weights have been used, and assuming that $g_{i \text { corr }}$ is $0.2 \mathrm{~d}^{-1} ;(\Delta)$ errors assuming $g_{i_{-} \text {corr }}$ is $2.0 ;(\nabla)$ errors assuming $g_{i_{-} \text {corr }}$ is $0.02 \mathrm{~d}^{-1}$. Short dashed lines indicate where $g_{i_{-} M R}=g_{i_{c} c o r r}$. To derive error when the proceeding stage (stage $i+1$ ) does not moult (i.e. C6 and in earlier stages when the original study indicates this is a non-moulter), we derive errors in stage $i$ associated with stage $i+1$ having a mean weight $50 \%$ greater than that at the point of entry to stage $i+1$ (indicated by ' $+50 \%$ '), a weight unchanged from that at entry ('0\%'), and a weight $50 \%$ less than that at the point of entry ('-50\%'). See text for details 


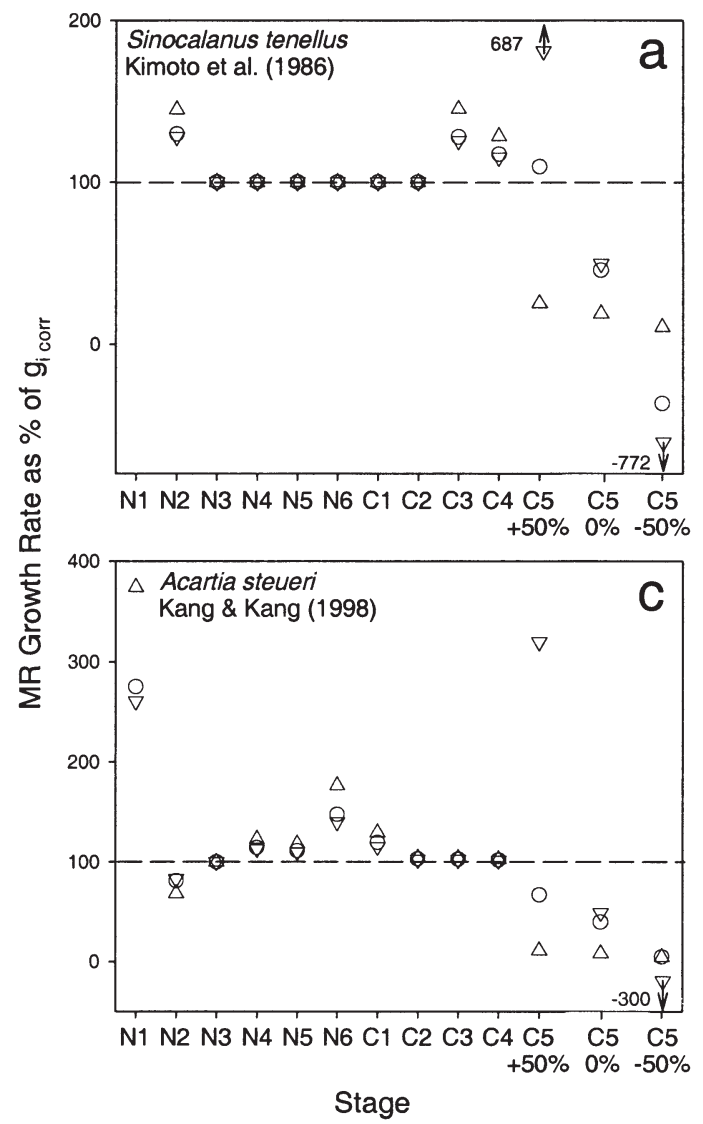

just $13.7 \%$ (Eurytemora affinis, Burkill \& Kendall 1982) to $47.2 \%$ of the correct growth rate. If the adult does grow (in our example, achieving a weight $50 \%$ greater than that at the point of moult), then in the vast majority of cases, C5 growth has still been dramatically underestimated. If the adult were to lose weight, then in most circumstances, the published growth values are even more erroneous than if body weight were constant (exceptions being Paracalanus/Clausocalanus spp. in Webber \& Roff 1995, and Centropages velificatus and Temora turbinata in Hopcroft et al. 1998). In the study of Shreeve et al. (2002), because C5 of Calanoides acutus and $\mathrm{C} 4$ of Rhincalanus gigas were not moulting, the MR growth rates of the $\mathrm{C} 4$ and $\mathrm{C} 3$ stages, respectively, are those in greatest error, with large underestimation having taken place. Assuming these non-moulting stages achieve a mean weight equal to that at entry to the stage, the $g_{i \_M R}$ values in their study are 22.6 and $11.1 \%$ of the correct growth values (taking a $g_{i \_c o r r}$ value of $0.2 \mathrm{~d}^{-1}$ ). In all these examples listed, we have given the error arising assuming $g_{i \_c o r r}$ to be $0.2 \mathrm{~d}^{-1}$. The degree of error is usually worse if this value is greater (as often it will be in warm waters), but better if growth is lower (see Fig. 5).

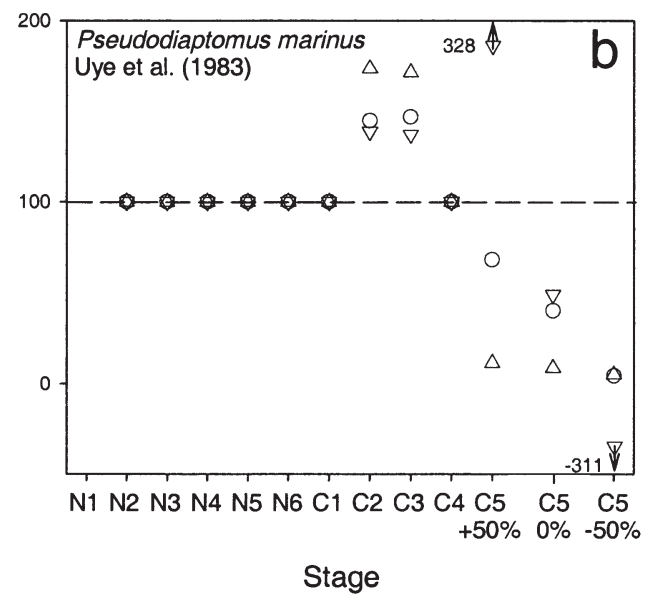

Fig. 6. Examination of errors associated with the application of the Moult Rate method to derive growth under laboratory conditions, using examples taken from the published literature. Symbols and derivation as given in Fig. 5. See text for details

Clearly, the most dramatic errors in growth arising from application of the MR method are in the C5 stage or other stages preceding a non-moulting stage. Underestimation of growth using the MR method can then be very large. In some cases, a growth value that is almost an order of magnitude too low has been calculated. The scenarios in Fig. 4 have been chosen to encompass a range of realistic situations; most show $g_{M R}$ to produce a substantial underestimation. Generally, only if both the nonmoulting stage is adding weight and the $g_{i_{-} \text {corr }}$ value is relatively low (i.e. $<<0.2 \mathrm{~d}^{-1}$ ), would $g_{M R}$ be an overestimate of $g_{\text {corr }}$. Satisfying both of these prerequisites is probably not common, and we might conclude that the MR method will most often produce gross underestimates of growth in stages that are followed by non-moulters.

The estimates of error described in Figs. $5 \& 6$ are only approximations as to the true error. In cases where both stages moult, we could only assess the errors arising from unequal stage durations. In those stages preceding a non-moulting stage, we have attempted to estimate error from both the stage duration of $i$ and taking various achieved mean weight scenarios for the non-moulting stage. Unfortunately, 
we simply do not have all the information necessary to account for the full degree of error. Nonetheless, our work is instructive as to where error arises, and as a first approximation of its size and sign. In our examples, if $g_{i \_c o r r}$ is not equal across consecutive moulting stages (Assumption 4 is violated), then $g_{i_{-} M R}$ may be in greater error than we have estimated. However, if $g_{i \_c o r r} / g_{i+1 \_c o r r}$ is greater than 1 , while $D_{i} / D_{i+1}$ is less than 1 , or vice-versa, error from each will tend to cancel. This is an important point, and this may act to cancel some of the error we have estimated. Often in later copepodite stages, $D_{i+1}$ may have a longer development time than $D_{i}$, but also a slower growth rate (see Fig. 3); these 2 parameters will to some degree cancel each other out. Although the errors are of opposite sign about ratios of 1 (Fig. 2), they are not symmetrical and opposite; hence, they do not entirely cancel when these 2 ratios multiplied together equal 1.

We have found only one instance of correct application of the MR approach for animals in a field situation (i.e. Shreeve \& Ward 1998). The most comprehensive examination of the MR equation to date is by ReyRassat et al. (2002). Examining Calanus helgolandicus in a laboratory experiment, they compared $g_{M R}$ growth rates with those obtained from weight at entry and exit from a stage and stage duration $\left(g_{\text {corr }}\right)$. They showed the latter to be better at demonstrating changes in growth resulting from food concentration, and the increase in lipid deposition through copepodite stages. In Rey-Rassat et al. (2002), errors in MR growth were attributed to the fact that 'the growth rate of 2 successive stages can vary significantly' (i.e. our Assumption 4 is violated). However, as we have shown here, even when consecutive stages have equivalent rates of growth, $g_{M R}$ can still be in gross error if development times are unequal (i.e. Assumption 3 is violated).

Where workers have derived secondary production, or indices or measures that rely upon MR growth, these will also be in error. Examples might include the growth versus body-weight (scaling) relationships of Peterson \& Hutchings (1995) and Richardson et al. (2001).

\section{Corrected equations (with and without mortality)}

There are several possible ways in which the MR equation can be corrected, but all must attribute changes in body weight to the correct time period. Stage-specific growth rates $\left(g_{i_{-} c o r r}\right)$ can be determined by measuring weight at the point of entry $\left(W_{i_{-} e n t r y}\right)$ and exit $\left(W_{i_{-} \text {exit }}\right)$ from a stage, as detailed in our Eq. (2), and referred to throughout this paper. Making measurements of such weights may be difficult, but if moulting rate is high, this is feasible. Shreeve \& Ward (1998) undertook this by measuring the weight of those individuals that moulted during their experiment, and these were assigned to appropriate stages as the weight at entry $\left(W_{i \_ \text {entry }}\right)$ and exit ( $\left.W_{\text {i_exit }}\right)$. Weights of animals after leaving the stage need to include the moulted exuviae in the $W_{i_{-} e x i t}$ term. These 2 weights are not affected by within-stage mortality; however, this approach, like many others, relies upon the accurate determination of stage development time. It is common for moulting rates from field experiments to be used to derive development time $\left(1 / M R_{i}=D_{i}\right)$. Such methods have been used not only in copepods, but in other crustaceans as well (e.g. euphausiids: Ross et al. 2000). This approach assumes a uniform age, and hence steady-state and zero within-stage mortality. Let us therefore start by exploring where age within stage is not uniform, but is rather simply altered by mortality, i.e there will be a greater number of younger animals in the stage than older animals.

Stage duration is determined by taking a collection of animals from the stage of interest, and incubating them for a period $L$. The proportion of animals which moult $(M)$ during this period is recorded, and expressed on a per day basis as moult rate $M R=M / L$. Stage duration $(D)$ is then estimated as $D=1 / M R$.

To examine the effect of within-stage mortality, let $f(x)$ denote the probability density function of time $(x)$ since moult $(0<x<D)$ of a randomly-selected animal in a given stage. For a given value of $x_{1}$ the animal will moult during the incubation period when $x+L>D$, i.e. with probability:

$$
M=\mathrm{P}(x+L>D)=\mathrm{P}(x>D-L)=\int f(x) \mathrm{d} x
$$

where the limits of integration are $D-L, D$.

(1) No mortality. If animals enter the stage at a uniform rate, the distribution of $x$ is uniform, i.e. $f(x)=$ $1 / D$. This gives:

$$
M=\int 1 / D \mathrm{~d} x=[D-(D-L)] / D=L / D
$$

i.e. $D=L / M$, and $D=1 / M R$. From here on, we term development obtained using this (zero mortality assumption) approach $D_{M R}$.

(2) Constant mortality rate. Mortality modifies the distribution of $x$. For a constant within-stage mortality rate $\left(\beta\left[\mathrm{d}^{-1}\right]\right)$, the expected number of animals surviving to time $x$ is proportional to $\exp (-\beta x)$ and the probability density of $x$ is given by:

$$
f(x)=\beta \exp (-\beta x) /[1-\exp (-\beta D)], 0<x<D
$$

This gives:

$$
M=\int f(x) \mathrm{d} x=\int \beta \exp (-\beta x) \mathrm{d} x /[1-\exp (-\beta D)]
$$

where the limits of integration are $D-L, D$. 


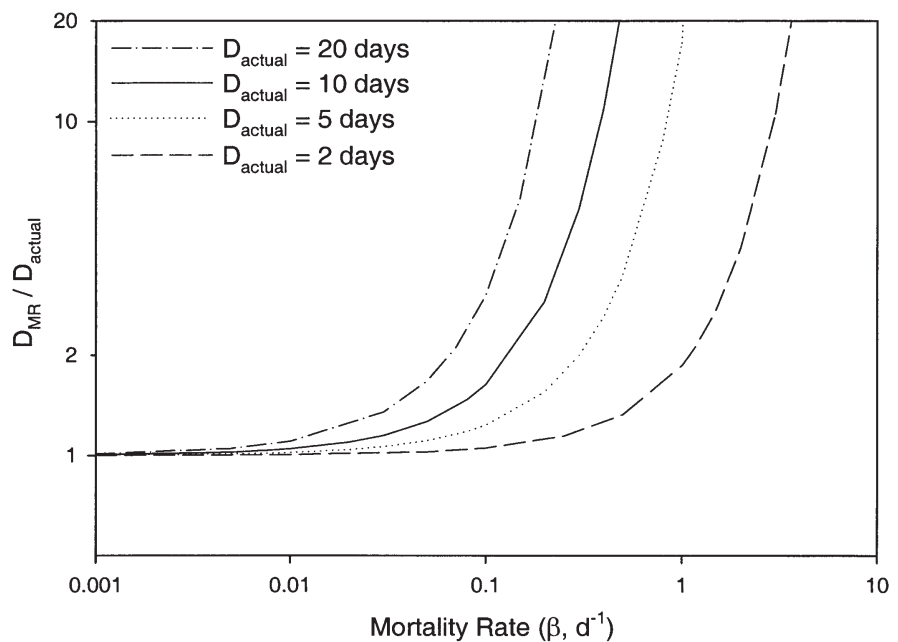

Fig. 7. Stage duration estimated by Moult Rate $\left(D_{M R}\right)$ in comparison to the true stage duration $\left(D_{\text {actual }}\right)$ as a function of mortality rate. Incubation period for MR determination $L$, is $1 \mathrm{~d}$

Evaluating the integral:

$$
M=\exp (-\beta D)[\exp (\beta L)-1] /[1-\exp (-\beta D)]
$$

and rearranging gives:

$$
\exp (-\beta D)=M /[M+\exp (\beta L)-1]
$$

Hence, a correct estimate for development time from the proportion moulting allowing for mortality is:

$$
D_{i \_a c t u a l}=\ln \left\{1+\left[\exp \left(\beta_{i} L_{i}\right)-1\right] / M_{i}\right\} / \beta_{i}
$$

To obtain this formulation, we have assumed that mortality ( $\beta$ ) acts in the field, but when we take these field animals and incubate them in the laboratory, mortality here is zero. The greater the rate of field mortality, the greater the degree of error will arise from $D_{M R}$. Fig. 7 examines how $D_{M R}$ diverges from the actual development time $\left(D_{\text {actual }}\right)$ as mortality increases. The larger the mortality rate, the more $D_{M R}$ overestimates the true stage duration. As incubation time $(L)$ approaches $D_{\text {actual, }}$ the less erroneous $D_{M R}$ becomes. This is why for a single incubation time $(L)$ long stage durations have greater error at any mortality value than short stage durations. We can compare these theoretical predictions with field data to establish the degree of error that might occur in nature. A compilation of individual stage durations as a function of temperature is given in Fig. 8a. The dashed line gives a regression through the data, while the solid line gives an approximation to the upper limits (drawn by eye). We then convert these values of development at any temperature to mortality rates that would produce $D_{M R}$ values that are twice $D_{\text {actual }}$. We do this for incubation periods of both 1 and 2 d. In Fig. 8b, these values (i.e. mortality rates producing a 2 -fold error in $D_{M R}$ ) are compared against a global data set of field mortality rates (from Hirst \& Kiørboe 2002). This comparison shows that mortality rates are infrequently high enough to cause a 2 -fold or greater error in average development times (given by the dashed line in Fig 8a). However, as development times approach their upper range (given by the solid line in Fig. 8a), field mortalities are often large enough to produce $D_{M R}$ values that overestimate $D_{\text {actual }}$ by 2 -fold or more. Incubating animals for longer, decreases the error in $\mathrm{D}_{M R}$ resulting from the effects of field mortality (Fig. 8b); however, increasing the incubation period will ultimately alter the animal's condition, its stage duration, and bring in other experimental artefacts. Mortality is not easy to measure, and therefore to allow for in this calculation, and yet as we have shown, it can have a profound impact on the estimation of development time from moult rate. One alternative is to not

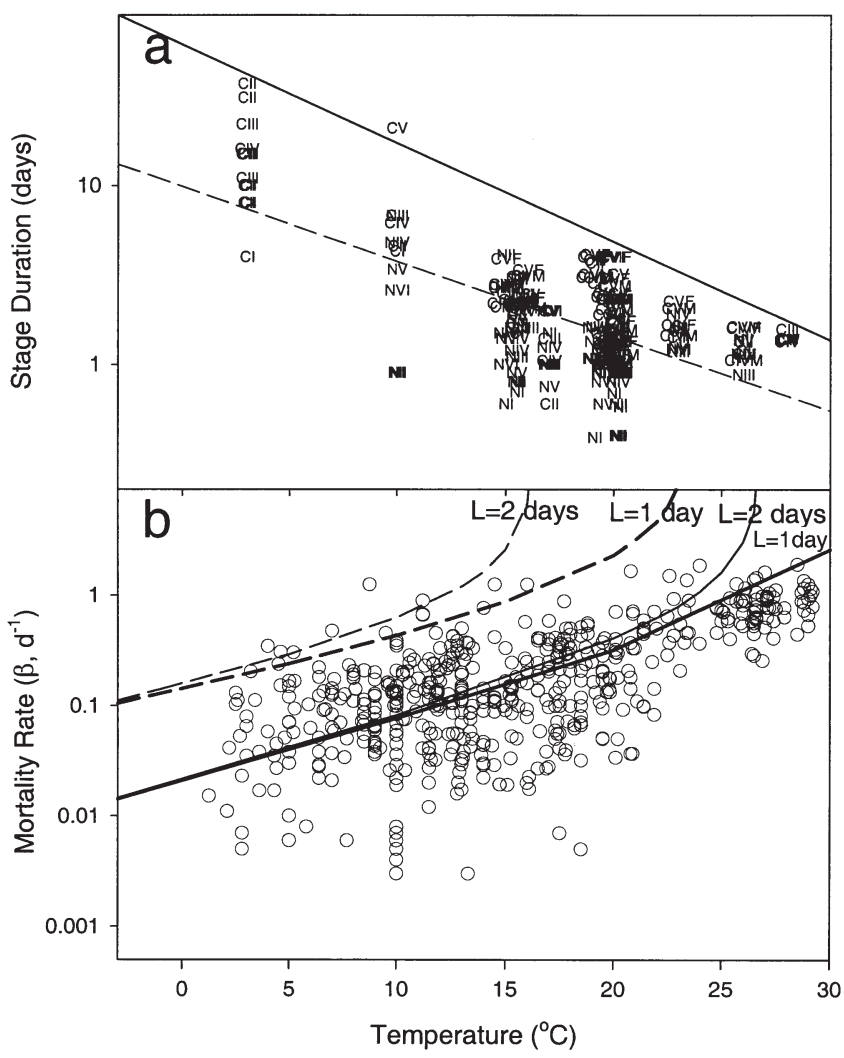

Fig. 8. Epi-pelagic copepod (a) individual stage duration $(D$ [d]) versus temperature of incubation. Dashed line: regression through data; solid line: upper limit to data. Stages are indicated. (b) Field mortality rates $\left(\beta\left[\mathrm{d}^{-1}\right]\right)$ versus environmental temperature (from Hirst \& Kiørboe 2002). Mortality values that would produce $D_{M R}$ values twice those of $D_{\text {actual }}$ are given for both the regression through the stage duration data (dashed line) and the upper limits to stage duration (solid line). Values are derived for 2 incubation lengths $(L)$ when measuring moult rate: $1 \mathrm{~d}$ (bold lines) and $2 \mathrm{~d}$ 
rely upon moult rate at all, if one can measure stage duration directly; for example, by following animals from entry to exit of a stage. The main problem then becomes that the longer we incubate an animal, the more it will diverge from natural rates. The implications of these findings may extend beyond copepods into other crustaceans, where development times have been derived from moulting (e.g. euphausiids and other crustaceans). We might conclude that many studies on copepod stage duration derived using the standard MR approach will have over-estimated stage duration. The MR equation commonly underestimates growth even when stage durations have been measured without error. When stage durations are overestimated (as now seems likely), growth will be even more severely underestimated.

We can combine the effects of mortality on stage duration to derive growth rates from the MR method when mortality acts. Eq. (4) becomes:

$$
g_{i_{-} \text {corr(mortality) }}=\ln \left(\frac{W_{i_{\_} \text {exit }}}{W_{i_{-} \text {entry }}}\right) \times \frac{1}{D_{i_{-} \text {actual }}}
$$

A second approach to determine growth is from the mid-point of stages $i$ to $i+1\left(g_{i \rightarrow i+1}\right)$. First, let us define this equation when there is no mortality:

$$
g_{i \rightarrow i+1}=\ln \left(\frac{\hat{W}_{i+1}}{\hat{W}_{i}}\right) \div\left[\left(D_{i_{-} \text {actual }}+D_{i+1 \_a c t u a l}\right) / 2\right]
$$

where $\hat{W}_{i}$ is the geometric mean weight of stage $i$, and $\hat{W}_{i+1}$ the geometric mean weight of stage $i+1$, including the weight of moult lost on transition between the stages. This equation exploits the fact that when growth is exponential and mortality is zero, the geometric mean weight represents that at the mid-time point of the stage. However, when mortality acts, this will alter the weight distribution such that the geometric mean weight will no longer represent that at the mid-time point through the stage duration. Furthermore, geometric mean weights are difficult to derive because weighing or elemental determination often involves the bulking of animals. By contrast, arithmetic mean weight can be determined even if animals are bulked in the weighing procedure. We therefore turn our attention to determining more practically achievable cases with equations that describe growth (with and without mortality) when arithmetic mean weights of consecutive stages are available.

The arithmetic mean weight of a sample of animals is given by:

$$
A W=\int W_{\text {entry }} \exp (g x) f(x) \mathrm{d} x
$$

where the limits of integration are 0 and $D_{\text {actual }}$ and $f(x)$ denotes the probability density function of time $x$ since moult of a randomlyselected animal.
(1) No mortality. In this case, $f(x)=1 / D_{\text {actual, }}$, so that:

$$
A W=\int W_{\text {entry }} \exp (g x) \mathrm{d} x / D_{\text {actual }}
$$

Evaluating the integral gives:

$$
A W=W_{\text {entry }}\left[\exp \left(g D_{\text {actual }}\right)-1\right] /\left(g D_{\text {actual }}\right)
$$

This is equivalent to Eq. (25) as presented by Kimmerer (1987). It can be written as:

$$
A W=W_{\text {entry }} \exp \left(g D_{\text {actual }} / 2\right) h_{0}\left(g_{1} D_{\text {actual }}\right)
$$

where the function $h_{0}\left(g, D_{\text {actual }}\right)=\left[\exp \left(g D_{\text {actual }} / 2\right)-\right.$ $\left.\exp \left(-g D_{\text {actual }} / 2\right)\right] /\left(g D_{\text {actual }}\right)$ measures the deviation from the weight mid-way through the stage.

For 2 successive stages $i$ and $i+1$, we have:

and:

$$
A W_{i}=W_{i \_ \text {entry }} \exp \left(g_{i} D_{i \_a c t u a l} / 2\right) h_{0}\left(g_{i}, D_{i \_a c t u a l}\right)
$$

$A W_{i+1}=W_{i+1 \_ \text {entry }} \exp \left(g_{i+1} D_{i+1 \_a c t u a l} / 2\right) h_{0}\left(g_{i+1}, D_{i+1 \_a c t u a l}\right)$

Since $W_{i+1 \_ \text {entry }}=W_{i_{-} \text {exit }}=W_{i_{-} \text {entry }} \exp \left(g_{i} D_{i \_ \text {actual }}\right)$, then:

$$
A W_{i+1}=W_{i \_ \text {entry }} \exp \left(g_{i} D_{i_{i} \text { actual }}\right) \exp \left(g_{i+1} D_{i+1 \_a c t u a l} / 2\right)
$$

Combining the above equations gives:

$\ln \left(A W_{i+1} / A W_{i}\right)=g_{i} D_{i \_a c t u a l}+g_{i+1} D_{i+1 \_a c t u a l}+\ln h_{0}\left(g_{i+1}\right.$, $\left.D_{i+1 \_a c t u a l}\right)-\ln h_{0}\left(g_{i,} D_{i \_a c t u a l}\right)$

The first 2 terms on the right-hand side correspond to the weighted average of the growth rates with weights proportional to the stage duration.

Assuming equal growth rates in successive stages, i.e. $g_{i}=g_{i+1}=g_{i \rightarrow i+1}$, we can obtain an equation for $g_{i \rightarrow i+1}$ as:

$$
\begin{gathered}
\left.\ln \left(A W_{i+1} / A W_{i}\right) /\left[\left(D_{i \_a c t u a l}+D_{i+1 \_a c t u a l}\right) / 2\right]=g_{i \rightarrow i+1}\right] \\
+\left[\ln h_{0}\left(g_{i \rightarrow i+1}, D_{i+1 \_a c t u a l}\right)-\ln h_{0}\left(g_{i \rightarrow i+1}, D_{i \_a c t u a l}\right)\right] / \\
{\left[\left(D_{i}+D_{i+1 \_a c t u a l}\right) / 2\right]}
\end{gathered}
$$

Eq. (22) can be solved through iteration to give $g_{i \rightarrow i+1}$. Hence, we have a new equation to describe growth using arithmetic mean weights and stage durations of consecutive (moulting) stages. This equation, however, assumes that within-stage mortality in the field is zero. If this is not the case, then the results will be in error; we shall return to the size of this error.

(2) Constant within-stage mortality. In this case, $f(x)=\beta \exp (-\beta x) /\left[1-\exp \left(-\beta D_{\text {actual }}\right)\right]$, and the arithmetic mean is given by:

$A W=\int W_{\text {entry }} \beta \exp [x(g-\beta)] \mathrm{d} x /\left[1-\exp \left(-\beta D_{\text {actual }}\right)\right]$

where the limits of integration are 0 and $\left.D_{\text {actual }}\right)$. Evaluating the integral gives:

$$
\begin{gathered}
A W=W_{\text {entry }} \beta\left\{\exp \left[D_{\text {actual }}(g-\beta)\right]-1\right\} / \\
\left\{[g-\beta]\left[1-\exp \left(-\beta D_{\text {actual }}\right)\right]\right\}
\end{gathered}
$$


or:

where:

$$
A W=W_{\text {entry }} \exp \left(g D_{\text {actual }} / 2\right) h\left(g, D_{\text {actual }} B\right)
$$

$$
\begin{gathered}
h\left(g, D_{\text {actualı }} \beta\right)=\beta\left\{\exp \left(g D_{\text {actual }} / 2-\beta D_{\text {actual }}\right)\right. \\
\left.-\exp \left(-g D_{\text {actual }} / 2\right)\right\} /\left\{[g-\beta]\left[1-\exp \left(-\beta D_{\text {actual }}\right)\right]\right\}
\end{gathered}
$$

This equation measures the deviation from the weight mid-way through moult. Our Eq. (25) is equivalent to Eq. (22) of Kimmerer (1987). For 2 successive stages $i$ and $i+1$, we have:

$$
\begin{gathered}
A W_{i}=W_{i \_ \text {entry }} \exp \left(g_{i} D_{i \_a c t u a l} / 2\right) h\left(g_{\mathrm{i}}, D_{\mathrm{i} \_a c t u a l}, \beta_{\mathrm{i}}\right) \\
A W_{i+1}=W_{i+1 \_ \text {entry }} \exp \left(g_{i+1} D_{i+1 \_a c t u a l} / 2\right) h\left(g_{i+1},\right. \\
\left.D_{i+1 \_a c t u a l}, \beta_{i+1}\right)
\end{gathered}
$$

Since $W_{i+1 \_ \text {entry }}=W_{i_{-} \text {exit }}=W_{i_{-} \text {entry }} \exp \left(g_{\mathrm{i}} D_{\mathrm{i} \_ \text {actual }}\right)$, then:

$$
A W_{i+1}=W_{i \_ \text {entry }} \exp \left(g_{i} D_{i \_a c t u a l}+g_{i+1} D_{i+1 \_a c t u a l} / 2\right) h\left(g_{i+1}\right. \text {, }
$$

Combining the above equations gives:

$$
\begin{aligned}
& \ln \left(A W_{i+1} / A W_{i}\right)=g_{i} D_{i \_a c t u a l}+g_{i+1} D_{i+1 \_a c t u a l} \\
& +\left[\ln h\left(g, D_{i+1 \_a c t u a l} \beta_{i+1}\right)-\ln h\left(g_{1} D_{i \_a c t u a l} \beta_{i}\right)\right]
\end{aligned}
$$

The first 2 terms on the right-hand side correspond to the weighted average of the growth rates in stages $i$ and $i+1$, with weights proportional to the stage duration.

Assuming equal mortality rate (i.e. $\beta_{i}=\beta_{i+1}=\beta_{i \rightarrow i+1}$ ), and equal growth rates in successive stages (i.e. $g_{i}=$ $\left.g_{i+1}=g_{i \rightarrow i+1}\right)$, gives:

$\ln \left(A W_{i+1} / A W_{i}\right) /\left[\left(D_{i \_a c t u a l}+D_{i+1 \_a c t u a l}\right) / 2\right]=g_{i \rightarrow i+1}+$ $\left[\ln h\left(g_{i \rightarrow i+1}, D_{i+1 \_a c t u a l}, \beta_{i \rightarrow i+1}\right)-\ln h\left(g_{i \rightarrow i+1}, D_{i \_a c t u a l}, \beta_{i \rightarrow i+1}\right)\right] /$

$$
\left[\left(D_{i \_a c t u a l}+D_{i+1 \_a c t u a l}\right) / 2\right]
$$

So, for given values of $\beta_{i \rightarrow i+1}, D_{i \_a c t u a l}$ and $D_{i+1 \_a c t u a l}$ and arithmetic mean weights of the consecutive stages (in stage $i+1$, the weight includes the moult lost at stage transition), the equation can be solved numerically for $g_{i \rightarrow i+1}$ using an iterative procedure. We have therefore produced an equation for growth between 2 arithmetic mean weights of consecutive stages when mortality is non-zero and known. Kimmerer (1987) compares arithmetic mean weights derived from Eqs. (25) \& (16) (his Eqs. 22 \& 25, respectively), and shows that the ratio of mean weights determined by the first and second of these is always $<1$; however, for reasonable values of growth and mortality, the ratio is never $<0.94$. We can compare results from both Eqs. (22) \& (31) under situations of mortality and no mortality. Our comparison is, therefore, slightly more complex than that of Kimmerer's (1987), in that we are examining not only the impact of mortality on mean weights, but on obtaining growth rates between these. Using reasonable durations of consecutive stages of 1 and $1.5 \mathrm{~d}$ ( $D_{\text {i_actual }}$ and
$D_{i+1 \text { actual }}$ respectively), arithmetic mean weights of 1 and $3 \mathrm{mg}$ ind. $^{-1}$, and a range of mortality rates of 0.0 to $1.0 \mathrm{~d}^{-1}$, that span most field estimates (see Fig. 8b) then the equation that assumes zero mortality (Eq. 22) gives a value of growth of $0.85 \mathrm{~d}^{-1}$, while the equation that considers mortality (Eq. 31) gives growth rates of 0.85 to $0.92 \mathrm{~d}^{-1}$. Using weights of 1 and $3 \mathrm{mg}$ ind. ${ }^{-1}$, stage durations of 10 and $15 \mathrm{~d}$ in consecutive stage pairs, and a range in mortality of 0 to $1.0 \mathrm{~d}^{-1}$, the equation that assumes zero mortality (Eq. 22) gives a value of growth of $0.085 \mathrm{~d}^{-1}$, while the equation that considers mortality (Eq. 31) gives growth rates of 0.085 to $0.11 \mathrm{~d}^{-1}$. In these cases, the equation that does not consider mortality (Eq. 22) gives growth values that are within $77 \%$ of the correct rate (determined using Eq. 31), even for (excessively) high mortality rates. These errors are clearly much less than those arising from the application of the original erroneous MR method as dealt with in the first part of this paper. Mortality is very difficult to measure, and hence we are left with a satisfactory result in this respect; we do not need to consider mortality when measuring growth using the modified methods, and can rely upon Eqs. (12) \& (22). However, the result is not entirely satisfactory in that we have used $D_{\text {actual }}$ values and not $D_{M R}$, and hence we are dependent upon stage durations being correct. Furthermore, as we have already demonstrated, the MR approach in determining stage duration $\left(D_{M R}\right)$ is very sensitive to mortality, and field mortality rates are often large enough and stage durations long enough, such that $D_{M R}$ may be a poor representation of true stage durations $\left(D_{\text {actual }}\right)$. Unless mortality is known to be reasonably low, and/or stage durations are relatively short (and $D_{M R}$ will approximate $D_{\text {actual }}$ ), then methods other than MR need to be considered in obtaining stage duration estimates.

The results from this paper are timely as we cannot continue to rely upon adult fecundity to measure secondary production in this important group (Hirst \& Bunker 2003). Juvenile copepod growth rates need to be measured directly and using accurate methods. The findings of this paper help us to consider how growth work should be planned and implemented in the future. We will clearly need to invest more effort in obtaining accurate stage durations.

Acknowledgements. A.G.H. was supported by the BAS DYNAMOE Programme and the NERC Marine Productivity Thematic Programme (Grant Refs. NER/T/S/1999/00057 and NE/C508418/1 to A.G.H.). This is contribution number 255 for the US GLOBEC Program. We wish to thank A. Atkinson and C. Miller for comments on an earlier draft. We are indebted to W. Kimmerer and anonymous referees who helped us greatly improve the clarity of this work. We thank those authors who supplied details of their own studies. 


\section{LITERATURE CITED}

Banse K (1995) Zooplankton: pivotal role in the control of ocean production. ICES J Mar Sci 52:265-277

Burkill PH, Kendall TF (1982) Production of the copepod Eurytemora affinis in the Bristol Channel. Mar Ecol Prog Ser 7:21-31

Diel S, Klein Breteler WCM (1986) Growth and development of Calanus spp. (Copepoda) during spring phytoplankton succession in the North Sea. Mar Biol 91:85-92

Durbin EG, Durbin AG, Campbell RG (1992) Body size and egg production in the marine copepod Acartia hudsonica during a winter-spring diatom bloom in Narragansett Bay. Limnol Oceanogr 37:342-360

Escribano R, Hidalgo P (2000) Influence of El Niño and La Niña on the population dynamics of Calanus chilensis in the Humbolt Current ecosystem of northern Chile. ICES J Mar Sci 57:1867-1874

Escribano R, McLaren I (1992) Testing hypotheses of exponential growth and size-dependent molting rate in two copepod species. Mar Biol 114:31-39

Escribano R, McLaren I (1999) Production of Calanus chilensis in the upwelling artea of Antofagasta, northern Chile. Mar Ecol Prog Ser 177:147-156

Escribano R, Marin VH, Hidalgo P (2001) The influence of coastal upwelling on the distribution of Calanus chilensis in the Mejillones Peninsula (northern Chile): implications for its population dynamics. Hydrobiologia 453/454: 143-151

Fransz HG, Diel S (1985) Secondary production of Calanus finmarchicus (Copepoda: Calanoidea) in a transitional system of the Fladen Ground Area (Northern North Sea) during the spring of 1983. In: Gibbs E (ed) Proc Eur Mar Biol Symp. Cambridge University Press, Cambridge, p 123-133

Fryd M, Haslund OH, Wohlgemuth O (1991) Development, growth and egg production of the two copepod species Centropages hamatus and Centropages typicus in the laboratory. J Plankton Res 13:683-689

Hart R (1990) Copepod post-embryonic durations: patterns, conformity, and predictability. The realities of isochronal and equiproportional development, and trends in the copepodid-naupliar duration ratio. Hydrobiologia 206: $175-206$

Hirst AG, Bunker AJ (2003) Growth of marine planktonic copepods: global rates and patterns in relation to chlorophyll a, temperature, and body weight. Limnol Oceanogr 48:1988-2010

Hirst AG, Kiørboe T (2002) Mortality of marine planktonic copepods: global rates and patterns. Mar Ecol Prog Ser 230:195-209

Hirst AG, McKinnon AD (2001) Does egg production represent adult female copepod growth? A call to account for body weight changes. Mar Ecol Prog Ser 223:179-199

Hopcroft RR, Roff JC, Webber MK, Witt DS (1998) Zooplankton growth rates: the influence of size and resources in tropical marine copepodites. Mar Biol 132:67-77

Huang C, Uye S, Onbé T (1993) Geographic distribution, seasonal life cycle, biomass and production of a planktonic copepod Calanus sinicus in the Inland Sea of Japan and its neighboring Pacific Ocean. J Plankton Res 15:1229-1246

Huntley ME, Lopez MDG (1992) Temperature-dependent production of marine copepods: a global synthesis. Am Nat 140:201-242

Hutchings L, Verheye HM, Mitchell-Innes BA, Peterson WT, Huggett JA, Painting SJ (1995) Copepod production in the southern Benguela system. ICES J Mar Sci 52:439-455
Jerling HL, Wooldridge TH (1991) Population dynamics and estimates of production for the calanoid copepod Pseudodiaptomus hessei in a warm temperate estuary. Estuar Coast Shelf S 33:121-135

Kang HK, Kang YJ (1998) Growth and development of Acartia steueri (Copepoda: Calanoid) in the laboratory. J Korean Fish Soc 31:842-851

Kimmerer WJ (1987) The theory of secondary production calculations for continuously reproducing populations. Limnol Oceanogr 32:1-13

Kimmerer WJ, McKinnon AD (1987) Growth, mortality, and secondary production of the copepod Acartia tranteri in Westernport Bay, Australia. Limnol Oceanogr 32:14-28

Kimoto K, Uye SI, Onbé T (1986) Growth characteristics of a brackish-water calanoid copepod Sinocalanus tenellus in relation to temperature and salinity. Bull Plankton Soc Jpn 33:43-57

Klein Breteler WCM, Fransz HG, Gonzalez SR (1982) Growth and development of four calanoid copepod species under experimental and natural conditions. Neth J Sea Res 16: 195-207

Liang D, Uye S (1991) Population dynamics and production of the planktonic copepods in a eutrophic inlet of the Inland Sea of Japan. IV. Pseudodiaptomus marinus, the eggcarrying calanoid. Mar Biol 128:415-421

Liang D, Uye S (1996a) Population dynamics and production of the planktonic copepods in a eutrophic inlet of the Inland Sea of Japan. II. Acartia omorii. Mar Biol 125: 109-117

Liang D, Uye S (1996b) Population dynamics and production of the planktonic copepods in a eutrophic inlet of the Inland Sea of Japan. III. Paracalanus sp. Mar Biol 127: 219-227

Liang D, Uye S, Onbé T (1996) Population dynamics and production of the planktonic copepods in a eutrophic inlet of the Inland Sea of Japan. I. Centropages abdominalis. Mar Biol 124:527-536

McKinnon AD (1996) Growth and development in the subtropical copepod Acrocalanus gibber. Limnol Oceanogr 41:1438-1447

McLaren IA, Tremblay JM, Corkett CJ, Roff JC (1989) Copepod production on the Scotian Shelf based on life-history analyses and laboratory rearings. Can J Fish Aquat Sci 46:560-583

Miller CB, Tande KS (1993) Stage duration estimation for Calanus populations, a modelling study. Mar Ecol Prog Ser 102:15-34

Miller CB, Johnson JK, Heinle DR (1977) Growth rules in the marine copepod genus Acartia. Limnol Oceanogr 22: 326-335

Miller CB, Huntley ME, Brooks ER (1984) Post-collection molting rates of planktonic, marine copepods: measurements, applications, problems. Limnol Oceanogr 29: $1274-1289$

Muxagata E, Williams JA, Sheader M (2004) Composition and temporal distribution of cirripede larvae in Southampton Water, England, with particular reference to the secondary production of Eliminius modestus. ICES J Mar Sci 61:585-595

Peterson WT (2001) Patterns in stage duration and development among marine and freshwater calanoid and cyclopoid copepods: a review of rules, physiological constraints, and evolutionary significance. Hydrobiologia 453/454:91-105

Peterson WT, Hutchings L (1995) Distribution, abundance and production of the copepod Calanus agulhensis on the Agulhas Bank in relation to spatial variations in hydrogra- 
phy and chlorophyll concentration. J Plankton Res 17: 2275-2294

Peterson WT, Tiselius P, Kiørboe T (1991) Copepod egg production, moulting and growth rates, and secondary production, in the Skagerrak in August 1988. J Plankton Res 13:131-154

Peterson WT, Gómez-Gutiérrez J, Morgan CA (2002) Crossshelf variation in calanoid copepod production during summer 1996 off the Oregon coast, USA. Mar Biol 141: 353-365

Rey-Rassat C, Irigoien X, Harris R, Head R, Carlotti F (2002) Growth and development of Calanus helgolandicus reared in the laboratory. Mar Ecol Prog Ser 238:125-138

Rey-Rassat C, Bonnet D, Irigoien X, Harris R, Head R, Carlotti F (2004) Seasonal production of Calanus helgolandicus in the Western English Channel. J Exp Mar Biol 313:29-46

Richardson AJ, Verheye HM (1998) The relative importance of food and temperature to copepod egg production and somatic growth in the southern Benguela upwelling system. J Plankton Res 20:2379-2399

Richardson AJ, Verheye HM (1999) Growth rates of copepods in the southern Benguela upwelling system: the interplay between body size and food. Limnol Oceanogr 44:382-3932

Richardson AJ, Verheye HM, Herbert V, Rogers C, Arendse LM (2001) Egg production, somatic growth and productivity of copepods in the Benguela Current system and Angola-Benguela front. S Afr J Sci 97:251-257

Richardson AJ, Verheye HM, Mitchell-Innes BA, Fowler JL, Field JG (2003) Seasonal and event-scale variation in growth of Calanus agulhensis (Copepods) in the Benguela upwelling system and implications for spawning of sar-

Editorial responsibility: Otto Kinne (Editor-in-Chief), Oldendorf/Luhe, Germany dine Sardinops sagax. Mar Ecol Prog Ser 254:239-251

Ross RM, Quetin LB, Baker KS, Vernet M (2000) Growth limitation in young Euphausia superba under field conditions. Limnol Oceanogr 45:31-43

Shreeve RS, Ward P (1998) Moulting and growth of the early stages of two species of Antarctic calanoid copepod in relation to differences in food supply. Mar Ecol Prog Ser 175:109-119

Shreeve RS, Ward P, Whitehouse MJ (2002) Copepod growth and development around South Georgia: relationships with temperature, food and krill. Mar Ecol Prog Ser 233: 169-183

Uye S, Iwai Y, Kasahara S (1983) Growth and production of the inshore marine copepod Pseudodiaptomus marinus in the central part of the Inland Sea of Japan. Mar Biol 73: 91-98

Verity PG, Smetacek V (1996) Organism life cycles, predation, and the structure of marine pelagic ecosystems. Mar Ecol Prog Ser 130:277-293

Vidal J (1980) Physioecology of zooplankton. I. Effects of phytoplankton concentration, temperature, and body size on the growth rate of Calanus pacificus and PseudoCalanus sp. Mar Biol 56:111-134

Walker DR, Peterson WT (1991) Relationships between hydrography, phytoplankton production, biomass, cell size and species composition, and copepod production in the southern Benguela Upwelling system in April 1988. S Afr J Mar Sci 11:289-305

Webber MK, Roff JC (1995) Annual biomass and production of the oceanic copepod community off Discovery Bay, Jamaica. Mar Biol 123:481-495

Submitted: June 17, 2004; Accepted: December 21, 2004 Proofs received from author(s): June 16, 2005 